The University of Southern Mississippi

The Aquila Digital Community

Faculty Publications

$5-1-1998$

\title{
Discrete-to-Continuum Simulation Approach to Polymer Chain Systems: Subdiffusion, Segregation, and Chain Folding
}

Grace M. Foo

National University of Singapore

Ras B. Pandey

University of Southern Mississippi, ras.pandey@usm.edu

Follow this and additional works at: https://aquila.usm.edu/fac_pubs

Part of the Physics Commons

\section{Recommended Citation}

Foo, G. M., Pandey, R. B. (1998). Discrete-to-Continuum Simulation Approach to Polymer Chain Systems: Subdiffusion, Segregation, and Chain Folding. Physical Review E, 57(5), 5802-5810.

Available at: https://aquila.usm.edu/fac_pubs/4971

This Article is brought to you for free and open access by The Aquila Digital Community. It has been accepted for inclusion in Faculty Publications by an authorized administrator of The Aquila Digital Community. For more information, please contact Joshua.Cromwell@usm.edu. 


\title{
Discrete-to-continuum simulation approach to polymer chain systems: Subdiffusion, segregation, and chain folding
}

\author{
Grace M. Foo ${ }^{1}$ and R. B. Pandey ${ }^{2}$ \\ ${ }^{1}$ Supercomputing and Visualization Unit, Computer Center, National University of Singapore, 119260 Singapore \\ ${ }^{2}$ Department of Physics and Astronomy, University of Southern Mississippi, Hattiesburg, Mississippi 39406-5046
}

(Received 17 December 1997)

\begin{abstract}
A discrete-to-continuum approach is introduced to study the static and dynamic properties of polymer chain systems with a bead-spring chain model in two dimensions. A finitely extensible nonlinear elastic potential is used for the bond between the consecutive beads with the Lennard-Jones $(\mathrm{LJ})$ potential with smaller $\left(R_{c}\right.$ $\left.=2{ }^{1 / 6} \sigma=0.95\right)$ and larger $\left(R_{c}=2.5 \sigma=2.1\right)$ values of the upper cutoff for the nonbonding interaction among the neighboring beads. We find that chains segregate at temperature $T=1.0$ with $R_{c}=2.1$ and remain desegregated with $R_{c}=0.95$. At low temperature $(T=0.2)$, chains become folded, in a ribbonlike conformation, unlike random and self-avoiding walk conformations at $T=1.0$. The power-law dependence of the rms displacements of the center of mass $\left(R_{\text {c.m. }}\right)$ of the chains and their center node $\left(R_{\mathrm{cn}}\right)$ with time are nonuniversal, with the range of exponents $\nu_{1} \simeq 0.45-0.25$ and $\nu_{2} \simeq 0.30-0.10$, respectively. Both radius of gyration $\left(R_{g}\right)$ and average bond length $(\langle l\rangle)$ decrease on increasing the range of interaction $\left(R_{c}\right)$, consistent with the extended state in good solvent to collapsed state in poor solvent description of the polymer chains. Analysis of the radial distribution function supports these observations. [S1063-651X(98)11205-9]
\end{abstract}

PACS number(s): 36.20.Ey, 02.70.Lq, 02.70.Ns, 83.10.Nn

\section{INTRODUCTION}

Development of molecular simulations using Monte Carlo and molecular dynamics methods, and their applications [16] to a variety of complex systems, have become a subject of considerable interest in teaching and research. In particular, computer simulation modeling has become an important tool to study the statics and dynamics of polymer chains in recent years $[5,6]$. One of the major problems in such simulations is the long relaxation time to reach steady-state and equilibrium configurations particularly for chains in melt, complex mixtures, and gel matrix. For example, the motion of a monomer (node) of a coarse-grained polymer chain in melts exhibits power laws, $R^{2} \sim t^{\nu}$ with different power-law exponents ( $\nu$ $=1,1 / 2,1 / 4,1 / 2,1)$ in various short to long time regimes $[7,8]$. Note that even the short time regime associated with Rouse-to-reptation crossover in polymer dynamics takes relatively large computational time steps [9-15]. It is rather difficult to cover all time regimes due to limitations on the computational resources. Extensive simulations $[5,6,9]$ are performed to examine the onset of reptation from short time Rouse dynamics [16] of a polymer chain in melt.

There are two main simulation approaches $[5,6,9]$ to study the statics and dynamics of polymer chains, i.e., lattice and off-lattice simulations to address appropriate questions. Offlattice simulations in a coarse-grained chain model deal with the motion of each monomer by a small amount. As a result, it is possible to take into account short time dynamics with good accuracy. It takes a considerably large number of time steps to study the long time behavior in a complex polymer melt. Using the discrete lattice approach, on the other hand, one may implement various dynamics, i.e., kink-jump, crank-shaft, and their combinations at various length scales, slithering-snake ("reptation") dynamics [5,6,9,17-20], etc., with relative ease. It is therefore possible to study the long time behavior with the help of appropriate dynamics. One should, however, be careful in implementing different local dynamics so as not to eliminate the desired physical properties arising from a short time local dynamics and introduce artificial effects. For example, if the "slithering-snake (reptation)" dynamics is used in conjunction with kink jump and crank shaft, then the reptation dynamics dominates over the short time Rouse behavior. Off-lattice simulations seem appropriate for short time local properties while the accelerated discrete simulation approaches seem to provide overall equilibrium properties. Since the discrete lattice simulations are generally more efficient for a long time (equilibrium) scale while the continuum (off-lattice) simulations are relatively slow but desirable to take into account the physics from the short time regime, we attempt to combine these methods in order to capture the appropriate effects.

\section{METHOD AND MODEL}

One of the common procedures in polymer simulations involves equilibrating the sample of the polymer chain and solvent and studying the statics and dynamics evolving from their statistical ensembles in which the constituents (chains) are in constant movement exploring their conformational phase space. How soon the system approaches its steadystate or equilibrium configurations depends on how fast the constituents are exploring their phase space. In complex systems like melts, movement of chain segments is very often too slow to reach equilibrium configurations in a desirable time. One then implements various dynamics to accelerate the process. The evolution of the system in statistical phase space could be thought of as a stirring process in which one may consider appropriate stirring pathways to bring such complex system close to a desirable state in phase space. In 
simulations, stirring of solution could be achieved in two steps: large scale stirring (global mixing-equilibration) by lattice simulation followed by small scale stirring (local mixing-equilibration) by off-lattice simulations.

To illustrate this method, we consider a two-dimensional system of size $L \times L$ as an example. In order to implement the lattice simulation we treat this space as a square lattice with unit lattice constant. Polymer chains, each of length $L_{c}$ consisting of $L_{c}+1$ nodes connected by unit bond length compatible with the lattice structure, are placed regularly on the lattice. Placing chains regularly in the beginning does not appear efficient at first glance, since we eventually like to have a randomly mixed configuration. However, we would be able to fill the lattice with arbitrary polymer concentrations (up to nearly $p=1$ ), which could otherwise be very time consuming in a random sequential process of introducing the chains in the system. Further in such a random sequential process of preparing the samples, the configurations of chains placed later are highly restricted (due to the presence of surrounding chains), which may result in biasedundesirable metastable states. A homogeneous distribution of chains may reduce the local inhomogeneity in the initial chain distribution. There could be other variant methods to introduce the chains for our system. After placing the chains, we reptate the chains for sufficiently long time steps to mix the system well so as to reduce or eliminate their memory. One may incorporate other dynamics such as kink jump and crank shaft, and may even involve temperature with appropriate interaction, however, we will restrict ourselves here to reptation dynamics alone in preparing the sample.

Reptating the chains accelerates the equilibration process. We would like to mention that reptation alone could lead to certain metastabilities as pointed out by several researchers $[19,20]$. However, the probability of such metastability is relatively low. Moreover, in our method, we implement other procedures (see below) that may help bring the chains out of such metastablity.

Now, we switch off the lattice structure and turn on the off-lattice simulation. We consider a coarse-grained beadspring model [21] for the polymer chain in which a finitely extensible nonlinear elastic (FENE) potential $\left(U_{F}\right)$ describes the bonded interaction between the consecutive beads of the chain, i.e.,

$$
U_{F}=-(k / 2) R^{2} \ln \left\{1-\left[\left(l-l_{0}\right) / R\right]^{2}\right\},
$$

where $l$ is the bond length, $R=l_{\max }-l_{0} . l_{0}, l_{\max }$, and $l_{\min }$ are the equilibrium value of the effective bond length, and its maximum and minimum values, respectively, such that $l_{\text {min }}$ $<l<l_{\max }$ and $l_{\text {min }}=2 l_{0}-l_{\max }$. We use $l_{\max }=1, l_{\text {min }}$ $=0.4$, and $l_{0}=0.7$. The associated spring constant $k$ is fixed at $k=10$. Next, we scale the bond length $l$ from its lattice value of one to a desirable value (closer to equilibrium bond length in off-lattice model, i.e., $\left\langle l_{0}\right\rangle \sim 0.7$. The lattice is also scaled simultaneously so that the chains are homogeneously distributed throughout the lattice. In addition we consider a Lennard-Jones (LJ) nonbonding interaction [1,2] between the neighboring beads except consecutive bonded beads which are held together by the FENE potential. The LJ interaction between two nodes $i$ and $j$ separated by $r_{i j}$ is described by

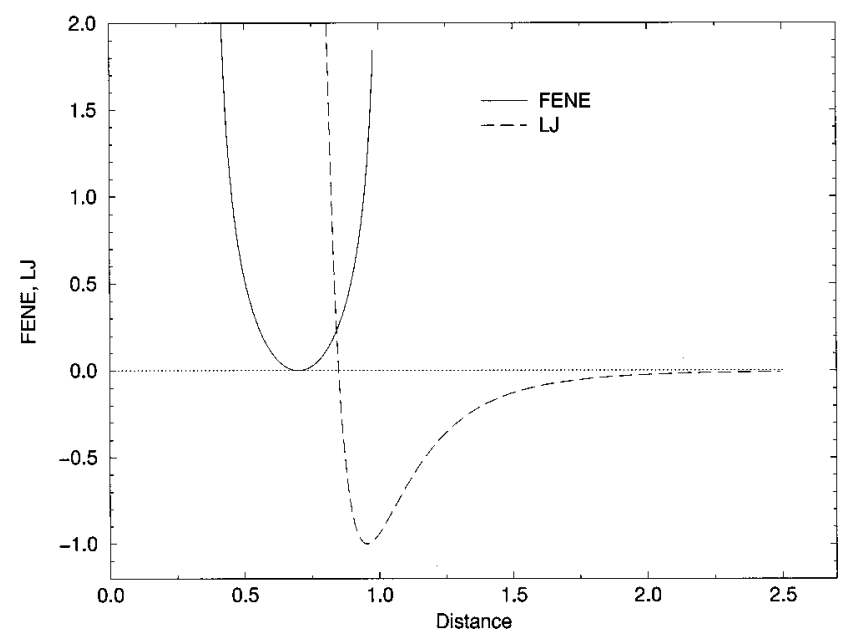

FIG. 1. Plot of FENE potential (dashed line) (for $K$ $\left.=10.0, l_{\min }=0.4, l_{\max }=1.0, l_{0}=0.7\right)$ and LJ potential $(\epsilon=1.0, \sigma$ $=0.85)$ vs distance.

$$
U_{\mathrm{LJ}}=4 \epsilon\left[\left(\frac{\sigma}{r_{i j}}\right)^{12}-\left(\frac{\sigma}{r_{i j}}\right)^{6}\right]
$$

where we select the parameters $\epsilon=1.0, \sigma=0.85$ in arbitrary unit. Spatial quantities such as rms displacements and radius of gyration are measured in arbitrary units. A typical variation of these potentials is shown in Fig. 1.

Using the Metropolis Monte Carlo algorithm [1-4] we attempt to move each bead by a small amount $[\delta r$ $=(\delta x, \delta y)]$ : find the energy $\left(U_{1}\right)$ of the bead in its original position and in the new position $\left(U_{2}\right)$, evaluate the difference $\delta U=U_{2}-U_{1}$, and accept the move with a Boltzmann distribution $\exp \left(-\delta U / k_{B} T\right)$. Attempting to move each bead once is defined as one Monte Carlo step (MCS). We move each node for a sufficiently long time to equilibrate the system before beginning to take the measurements. The number of time steps needed to achieve equilibration is now reduced considerably with reptation of chains in discrete lattice space than without it. We would like to point out that such an athermal reptation (preinitial stirring) corresponds to a high temperature equilibration. Switching to an off-lattice thermal simulation at a temperature $(T)$ then implies a quenching, the depth (drop in temperature) of which depends on $T$. In our simulation (see below), we have tried to approach an equilibrium-steady state where the temporal variation of bond length and radius of gyration approaches their stable values. We have not identified the theta temperature in this study although we have studied some effect of temperature. In the following we present some of our data for the conformation and dynamics of chains.

\section{RESULTS}

Most simulations are performed on a $100 \times 100$ space, although different sample sizes were also used to check for severe finite size effects. Low $(T=0.20)$ and high $(T=1.0)$ temperatures (in unit of $\epsilon / k_{B}$ with the Boltzmann constant $k_{B}$ ) are considered to see the contrast between the evolution of stable conformations and dynamics, with two upper cutoffs $\left(R_{c}=0.95,2.1\right)$ of the nonbonding potentials on chains 
(a)
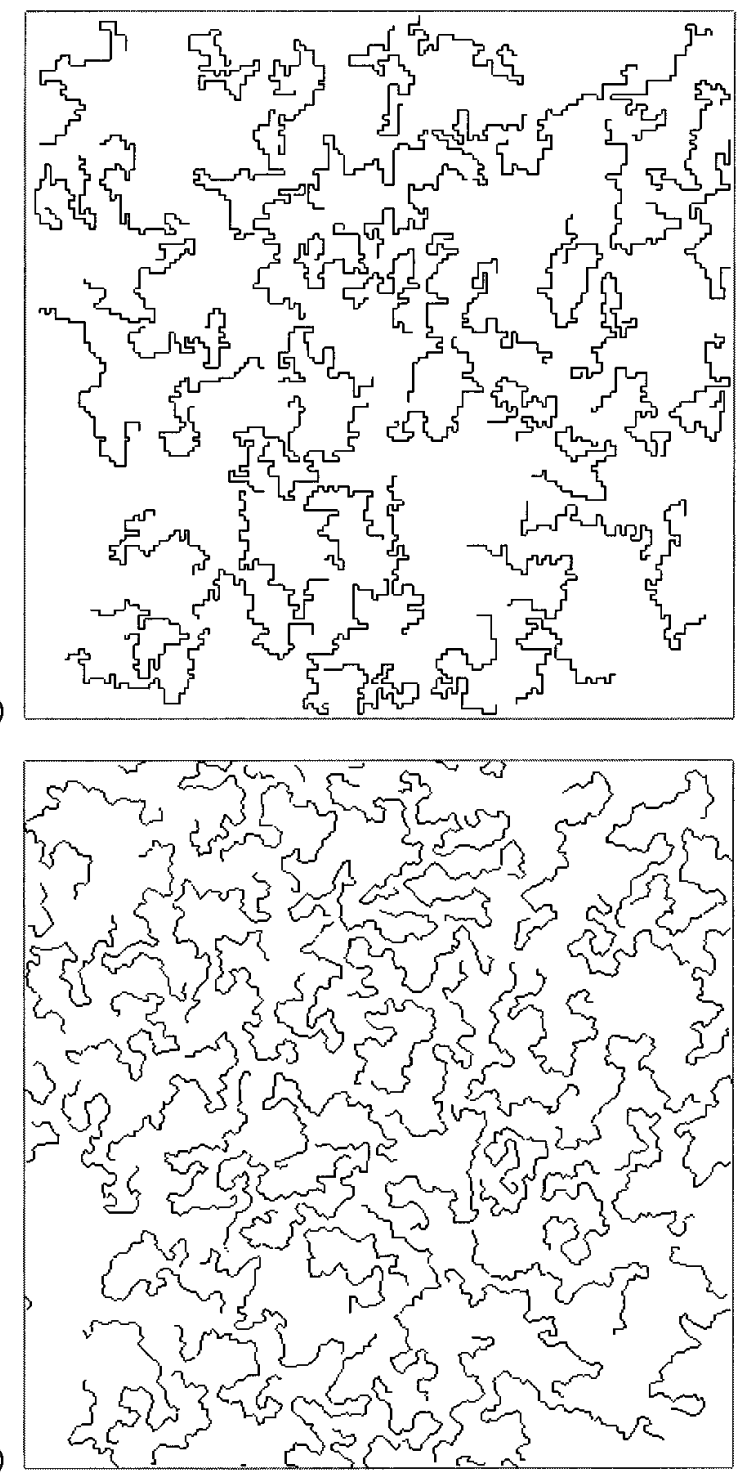

(c)

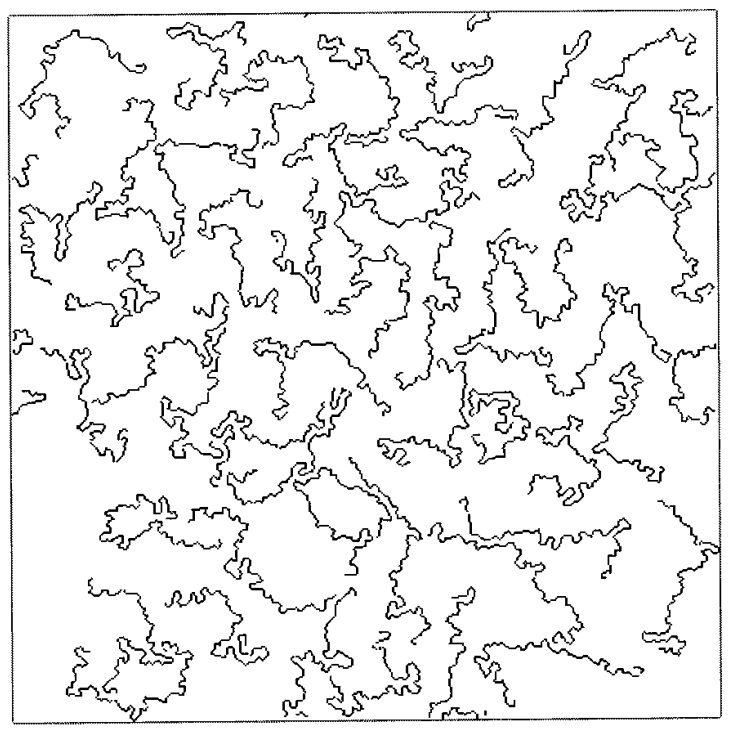

(d)

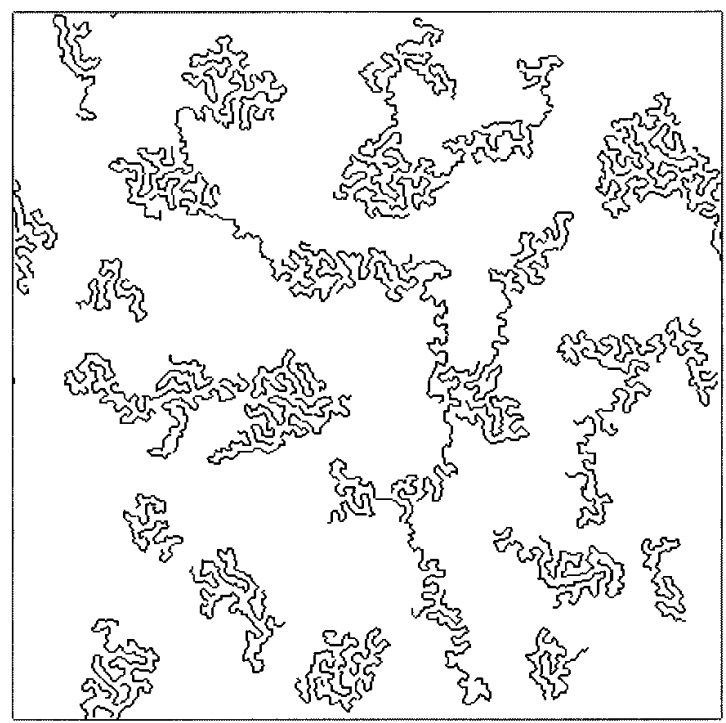

(e)

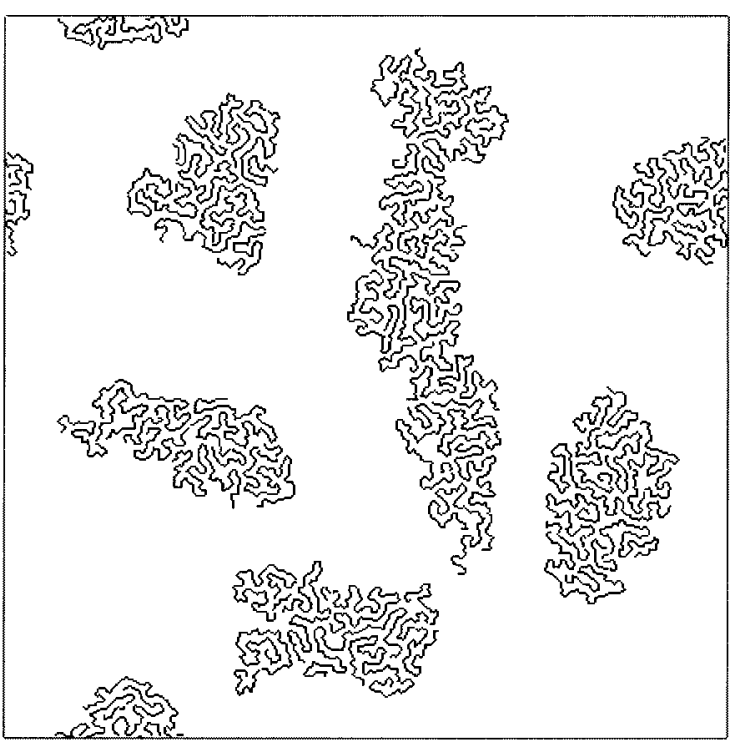

FIG. 2. Snapshots of chains of length $L_{c}=80$ with the upper cutoff $R_{c}=2.1$ of the LJ potential at $T=1.0$ and $p=0.4$ at various time steps: (a) just after large scale stirring (reptation on discrete lattice for $t=40000$ steps), (b) initial configuration after a small-scale mixing (node movement for $t=20000$ steps) and thereafter at $t=2^{10}(\mathrm{c}), t=2^{16}(\mathrm{~d}), t=2^{20}$ (e) steps. 
(a)
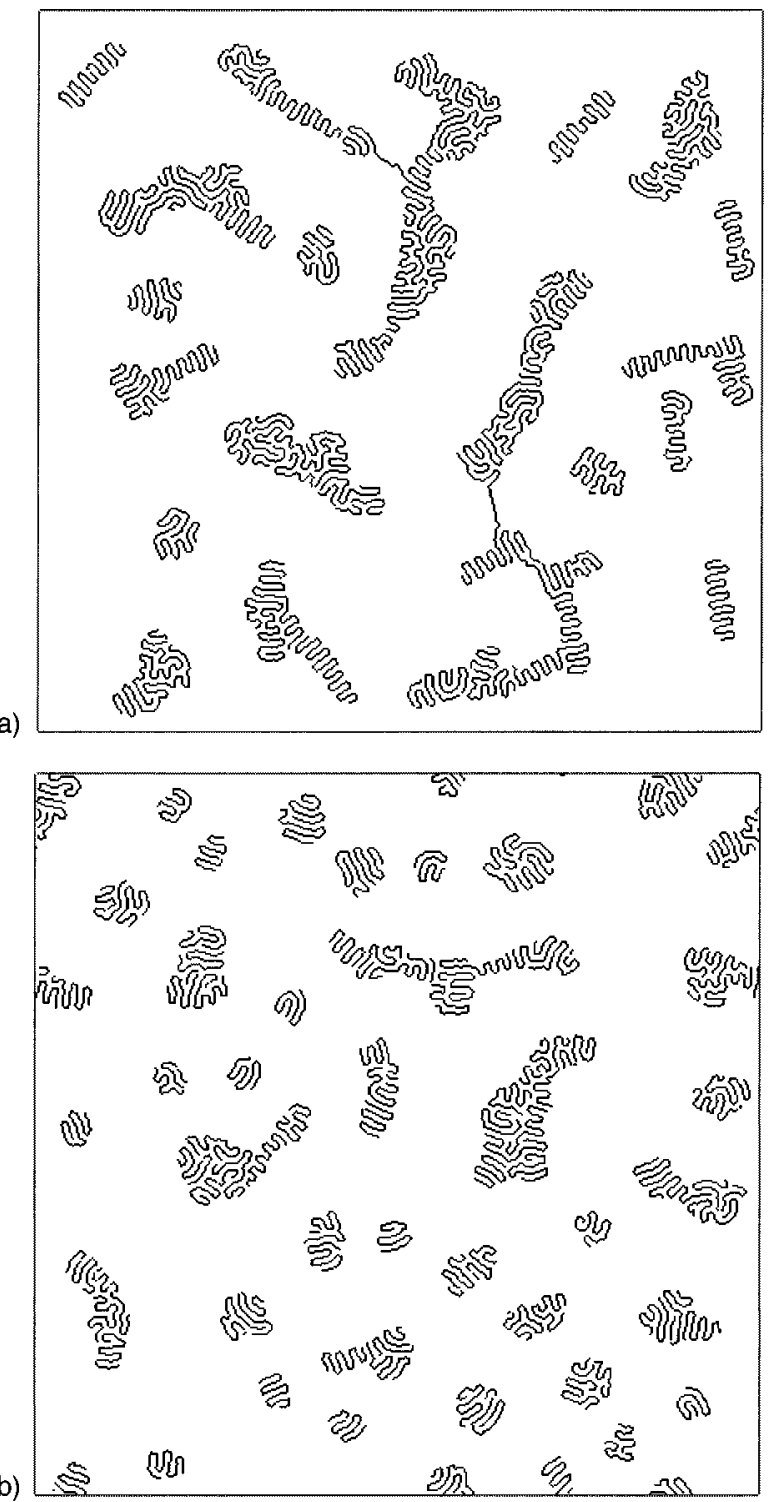

FIG. 3. Snapshots of the chains at $t=2^{20}$ steps for $L_{c}=80$ (a) and $L_{c}=40$ (b) at $p=0.4, T=0.2$, with upper cutoff $R_{c}=2.1$.

of length $L_{c}=20,40,60$, and 80 at polymer concentrations $p=0.05,0.2$, and 0.4 . We have used 8-10 independent samples (different initial configurations) for each set of data.

\section{A. Segregation, desegregation, and chain folding}

Distribution of polymer chains and their conformations at various stages of their evolution are studied by visual inspection of the snapshots. Figure 2 shows a typical evolution at $p=0.4$ and $T=1.0$ with $R_{c}=2.1$. From Fig. 2(a), we see that the chains are uniformly distributed throughout the system and their conformations appear to be random with excluded volume constraints [self-avoiding walk (SAW)]. The lattice constraints are visible with a constant discrete bond length and their orientations constrained to the square lattice. With a small local mixing (switching on the off-lattice simulation) [Fig. 2(b)], the lattice discreteness of the chains' conformations reduces considerably. After 1024 MCS [Fig. 2(c)], chains begin to segregate and form aggregates as the interactions begin to affect their mobility. At 65536 step [Fig.

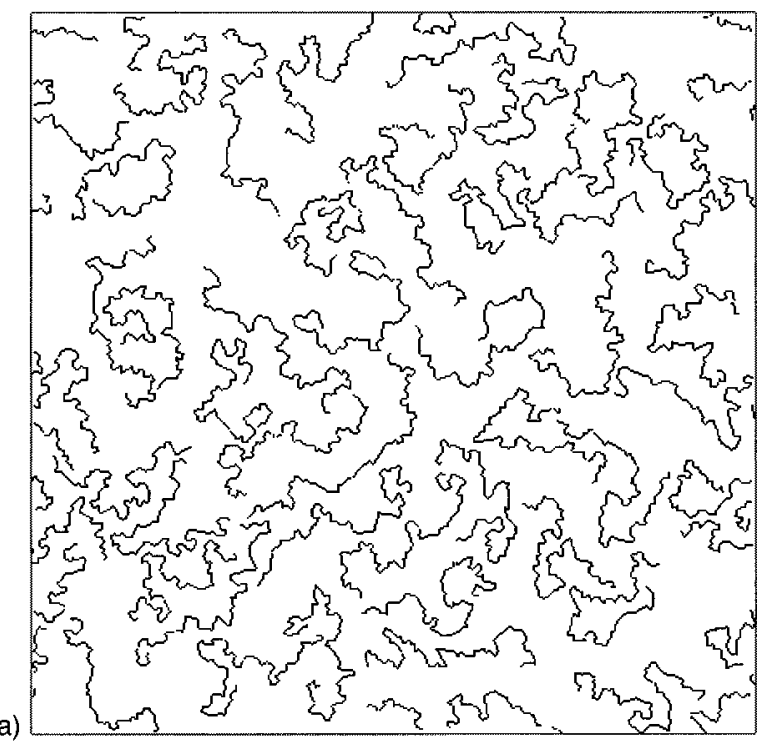

(a)

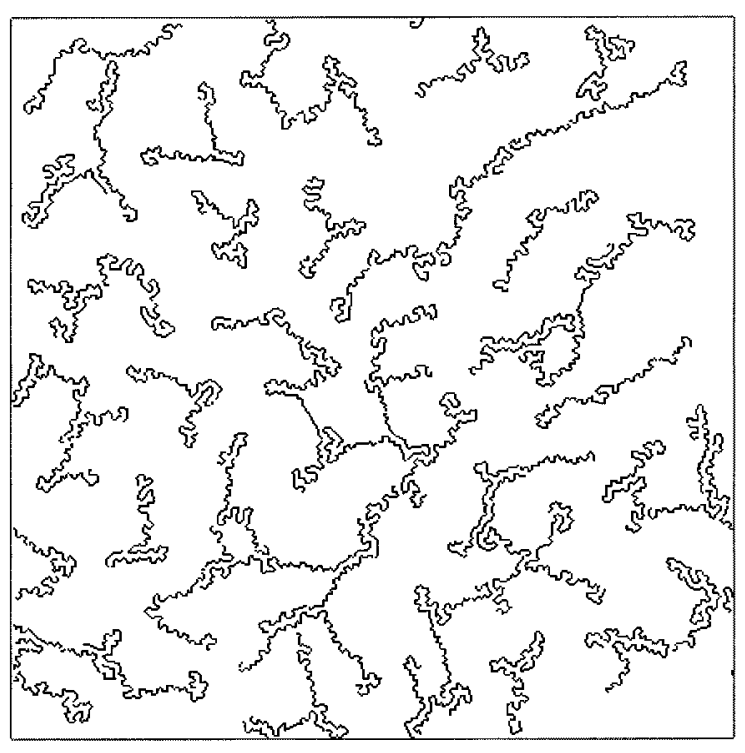

FIG. 4. Snapshots of the chains with the upper cutoff $R_{c}$ $=0.95$ for $L_{c}=80, p=0.4$ at $T=1.0$ (a) and 0.2 (b).

$2(d)]$ the segregations of chains into clusters of chain aggregates becomes pronounced, which finally develops into larger aggregates of chains at 1048576 step [Fig. 2(e)]. Note that the growth of the clusters (aggregates) becomes very slow now, as the mobility of chains becomes very low (see below). Chains are bounded together by the nonbonding $(\mathrm{LJ})$ interaction, which is dominant as the chain nodes approach closer within the attractive cutoff range $\left(R_{c}=2.1\right)$ at this temperature $(T=1.0)$. Such a segregation of chains in two dimensions has been studied before $[5,9]$.

A similar simulation at a low temperature $(T=0.2)$ shows a spectacular evolution of the chains' distribution and their conformations shown in Fig. 3. Note the contrast and difference in conformation of chains at low temperature (Fig. 3) from that at temperature $T=1.0$ [Fig. 2(e)] at the same time starting from the same initial configuration in a discrete stirring state [Fig. 2(a)]. Chains are folded in a rather uniform fashion unless hindered by clustering. We are not aware of such conformational change, i.e., from random conformations at $T=1.0$ to folded coil (ribbonlike) conformations in 


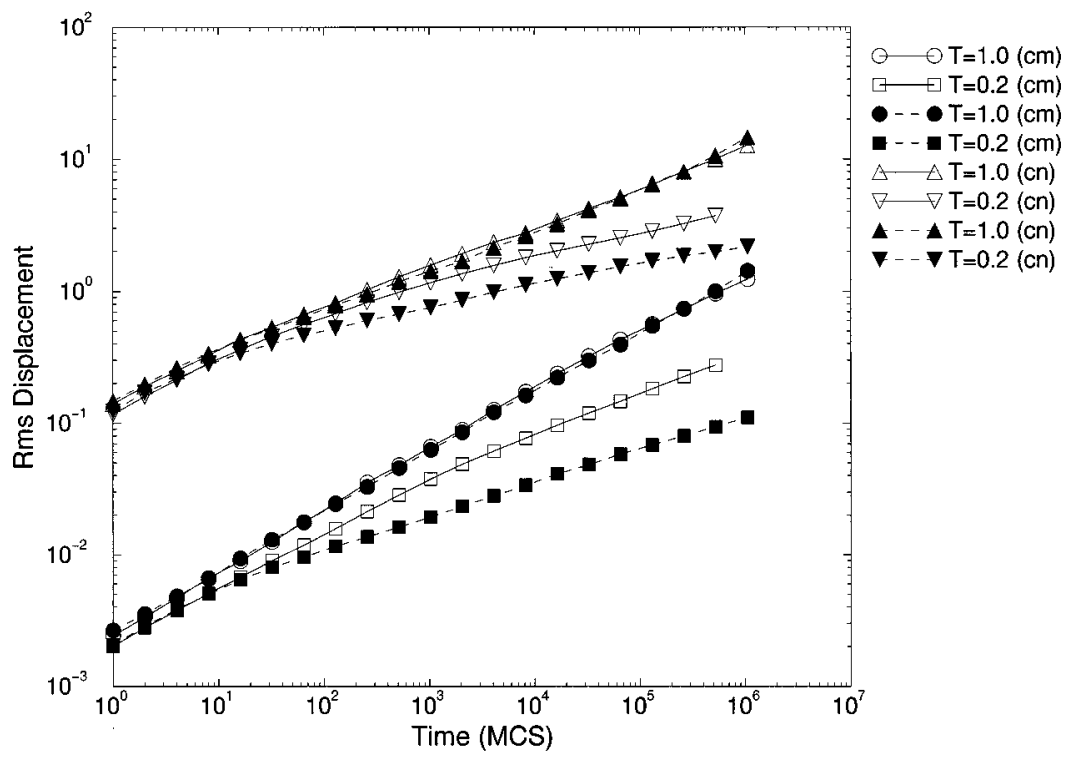

FIG. 5. RMS displacement for the center of mass of the chains (c.m.) and their center nodes (cn) vs time on a log-log scale for $L_{c}$ $=40$ and $p=0.4$, with $R_{c}=2.1$ (open) and 0.95 (filled).

two dimension at low temperatures $(T=0.2)$. We further note that such a spectacular conformational phase transition persists even with smaller chain sizes [compare Figs. 3(a) and $3(\mathrm{~b})]$.

As mentioned above, we have carried out simulations also with a relatively small value of the upper interaction cutoff, i.e., $R_{c}=2^{1 / 6} \sigma=0.95$ of the LJ interaction. Such a shortrange interaction brings our model closer to chains of beads with only hard-core (excluded volume sphere) interaction. The above characteristic conformational phase transition from random conformation to folded-coil conformation on lowering the temperature from $T=1.0$ to $T=0.2$ during segregation is also observed here (see Fig. 4). However, with the lower range of interaction at the low temperature, the size of the coil, i.e., the amplitude of folding, has reduced considerably. The ramification in the random conformations is more pronounced at a smaller scale.

Unlike the chains with relatively longer range interactions [i.e., with $R_{c}=2.1$, Fig. 2(e)], we do not observe segregation of chains with shorter range of interaction $\left[R_{c}=0.95\right.$, Fig. 4(a)]. Segregation of chains and formation of stable aggregates [Fig. 2(e)] thus depends on two factors: (i) mobility (which is higher at higher temperature), and (ii) the interactions (the longer the range of attractive $\mathrm{LJ}$ interaction and its magnitude, the more stable is the aggregate). Despite the mobility at $T=1.0$, the range of interaction $R_{c}=0.95$ is too small to form an aggregate within our observation time.

\section{B. RMS displacements}

During the structural evolution we calculate the rms displacement of the center node $\left(R_{\mathrm{cn}}\right)$ and the center of mass $\left(R_{\text {c.m. }}\right)$ of the chain periodically. The average rms displacement per chain at time $t$ is defined as

$$
R_{\mathrm{rms}}(t)=\sqrt{\left\langle x^{2}(t)\right\rangle+\left\langle y^{2}(t)\right\rangle},
$$

where

$$
\begin{aligned}
& \left\langle x^{2}(t)\right\rangle=\frac{1}{n_{c} n_{s}} \sum_{m=1}^{n_{s}} \sum_{k=1}^{n_{c}} x_{k m}^{2}, \\
& \left\langle y^{2}(t)\right\rangle=\frac{1}{n_{c} n_{s}} \sum_{m=1}^{n_{s}} \sum_{k=1}^{n_{c}} y_{k m}^{2},
\end{aligned}
$$

where $x(t)$ and $y(t)$ are the $x$ and $y$ components of the displacement of chains at time $t, n_{c}$ is the number of chains, and $n_{s}$ is the number of samples. Attempts are made to find the leading power-law dependence of these displacements with time described by exponents $\nu_{1}$ and $\nu_{2}$, i.e.,

$$
\begin{gathered}
R_{\mathrm{c} . \mathrm{m} .} \sim t^{\nu_{1}}, \\
R_{\mathrm{cn}} \sim t^{\nu_{2}} .
\end{gathered}
$$

A typical variation of rms displacements with time is presented in Fig. 5. The visual inspection of these plots suggests a relatively good power-law dependence in the long time regime. Chains have traveled a distance of the order of their radius of gyration and larger. The rms displacement for the center of mass (c.m.) of the chains is normalized by $L_{c}$ (the size of the chain); therefore it appears smaller than corresponding values for the center node $(\mathrm{cn})$. In fact, it is larger. The slope of the least square fit of these data points may provide an estimate of the exponents $\nu_{1}$ and $\nu_{2}$. However, one may resort to a better resolution by evaluating the slopes at regular intervals of time. In other words, we may evaluate the slopes of a set of consecutive data points (say 2, 3, or more) throughout the interval to see the trend. Figure 6 shows a typical plot of these power-law exponents versus time for $L_{c}=40$ chains at $p=0.40$. Obviously, the magnitude of the exponents is fluctuating and the fluctuations could be improved by increasing the number of statistics as we have observed. Nevertheless, it is possible to analyze the dynamics of polymer chains and their nodes, and find the trend as the system evolves. From these data (Fig. 6), we see a dis- 

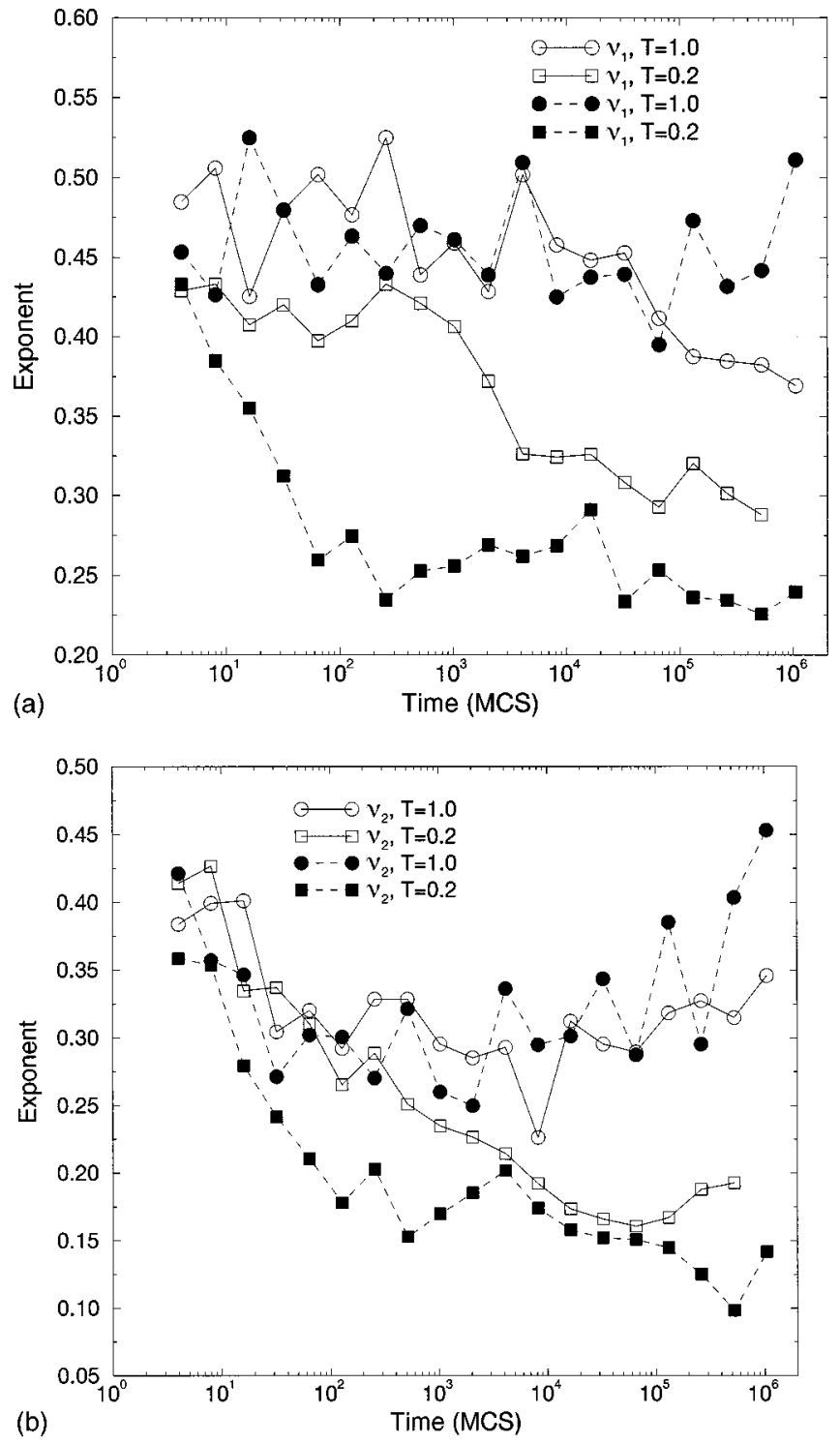

FIG. 6. Variation of the instantaneous exponent for the rms displacement of the center of mass (a) and center node (b) for $L_{c}$ $=40, p=0.4$ with $R_{c}=2.1$ (open) and 0.95 (filled).

tinct difference in the magnitude of $\nu_{1}$, the power-law exponent for the center of mass of the chains, at low and high temperatures $(T=0.20,1.0)$ in long time regime. For example, with the range of interaction $R_{c}=2.1$, the magnitude of $\nu_{1}$ drops from about 0.4 at $T=1.0$ to about 0.30 at $T$ $=0.2$. The difference in the magnitude of $\nu_{1}$ increases $\left(\nu_{1}\right.$ $\simeq 0.45$ at $T=1.0$ to $\nu_{1} \simeq 0.25$ at $T=0.2$ ) with the smaller range of interaction, $R_{c}=0.95$. Although the data points seem to have large fluctuations at this scale, the difference in magnitude of $\nu_{1}$ is larger than the range of fluctuations, a measure of the error bar.

Thus, we see that the temperature affects the power-law behavior of the center of mass of the chains in an important fashion leading to a nonuniversal power law (exponent). The range of interaction also seems to affect the magnitude of the power-law exponents. However, the difference in magnitude of $\nu_{1}$ due to range of interaction is small and seems less reliable due to lack of adequate quality data. The effect of

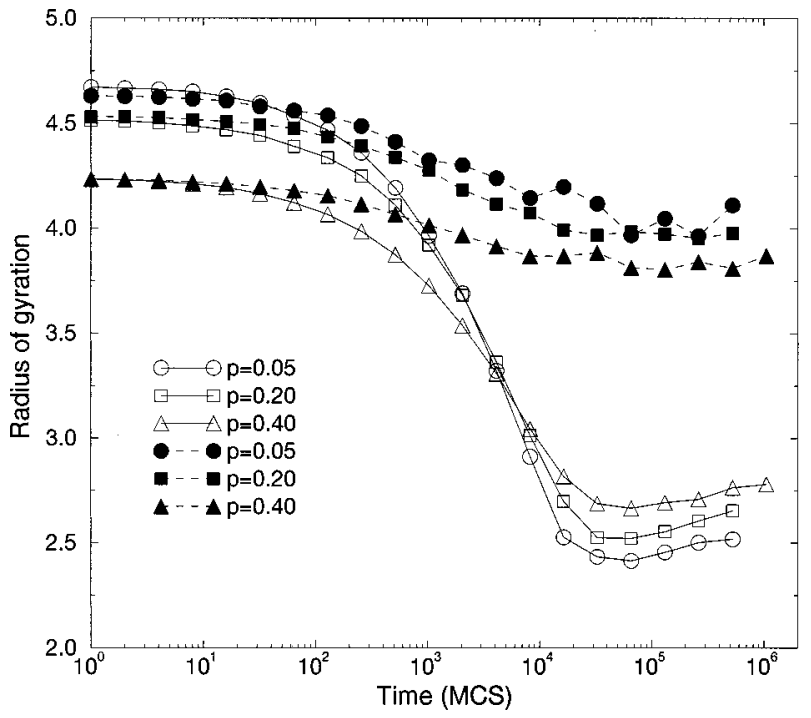

FIG. 7. Typical evolution of the radius of gyration $\left(R_{g}\right)$ with time for $L_{c}=40, T=1.0$ with $R_{c}=2.1$ (open) and 0.95 (filled).

temperature in controlling the power-law behavior of the center node of the chains is also quite clear: $\nu_{2} \simeq 0.3$ at $T=1.0$ drops down to $\nu_{2} \simeq 0.10$ at $T=0.2$ [see Fig. 6(b)]. However, the effect of the range of interaction on the powerlaw behavior of the center node (i.e., $R_{\mathrm{cn}} \sim t^{\nu_{2}}$ ) is not as clear again due to lack of sufficient data.

In the following we describe some other estimates of the exponents $\left(\nu_{1}\right.$ and $\left.\nu_{2}\right)$ as examples to gain insight into the range of error bars and effects of chain length and polymer concentration. With the upper cutoff, $R_{c}=2.1$, in the short time regime, we find $\nu_{1} \simeq 0.45 \pm 0.07$ and $\nu_{2} \simeq 0.30 \pm 0.06$ at $T=1.0$ for $L_{c}=80$ chains at $p=0.2$. For $L_{c}=40$, at $T$ $=0.2, \quad \nu_{1} \simeq 0.4$ (short time) to $\nu_{1} \simeq 1 / 4$ (long time) and $\nu_{2}$ $\simeq 1 / 4$ to $\nu_{2} \simeq 1 / 10$. For $L_{c}=80, T=0.2, \nu_{1} \simeq 0.4$ (short time) to 0.3 (long time), and $\nu_{2} \simeq 0.4$ (short time) to 0.1 (long time) with a continuous decay. The values of these exponents $\left(\nu_{1}, \nu_{2}\right)$ in the short time regime are given only to see the

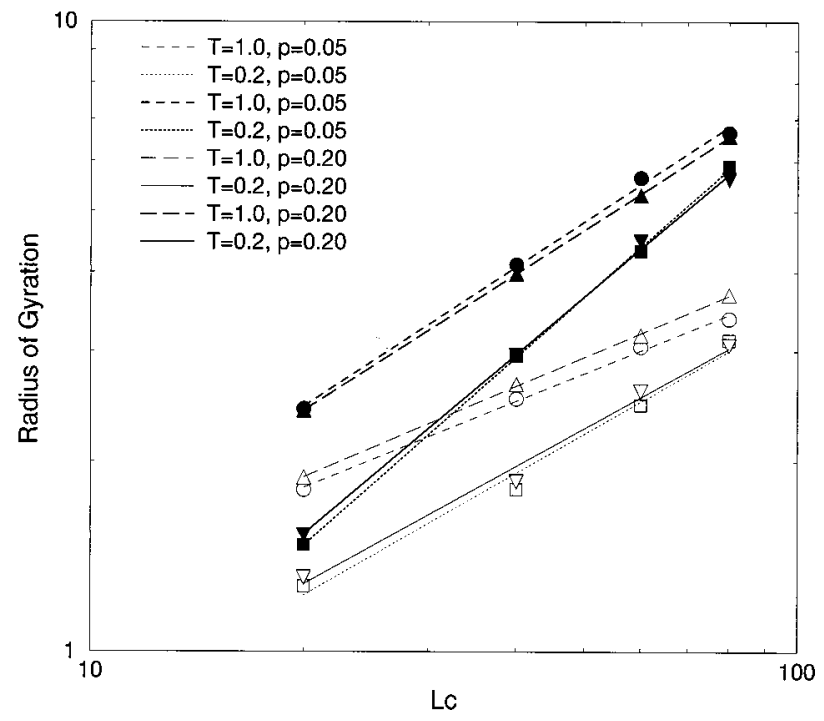

FIG. 8. Variation of $R_{g}$ vs $L_{c}$ on a log-log scale for chains at $p=0.05,0.2, T=0.2,1.0$ with $R_{c}=2.1$ (open) and 0.95 (filled). 
TABLE I. Exponent $\gamma$ in $R_{g} \sim L_{c}^{\gamma}$. Errors on the order $\sim 0.04$.

\begin{tabular}{lccc}
\hline \hline Polymer concentration & Temperature & \multicolumn{2}{c}{$\gamma$} \\
$p$ & $T$ & $R_{c}=2.1$ & $R_{c}=0.95$ \\
\hline 0.05 & 1.0 & 0.46 & 0.74 \\
0.2 & 1.0 & 0.48 & 0.72 \\
0.4 & 1.0 & & 0.70 \\
0.05 & 0.2 & 0.64 & 0.99 \\
0.2 & 0.2 & 0.62 & 0.95 \\
0.4 & 0.2 & & \\
\hline \hline
\end{tabular}

trend, and not to confuse with the chain dynamics in the asymptotic power-law regime since the system is not in equilibrium in short time regimes. Small values of the exponent in long time, however, implies that the chains and nodes are relatively less mobile as the segregation leads to stable or metastable aggregates of clusters at $T=1.0$. This is consistent with our visual analysis of the evolution of chains conformation and their aggregates in the preceding section.

\section{Radius of gyration and bond length}

A typical evolution of the radius of gyration $\left(R_{g}\right)$ in time is shown in Fig. 7. Note that $R_{g}$ has approached to a constant (saturated) value in the long time regime. This is an indication that our system has reached a steady-state-equilibrium as far as the conformation of chains is concerned. We see that the equilibrium value of $R_{g}$ depends on the value of upper interaction cutoff $\left(R_{c}\right)$. At $T=1.0$ with $R_{c}=2.1, R_{g}$ seems to increase on increasing $p$ from 0.05 to 0.4 . The radius of gyration appears to be less sensitive to polymer concentration with the smaller interaction cutoff $\left(R_{c}=0.95\right)$; however, our data show a decreasing trend of $R_{g}$ with increasing $p$. Thus, the polymer concentration affects the magnitude of $R_{g}$ differently with the smaller and larger interaction cutoffs. Further, we find that increasing the range of interaction $R_{c}$ from 0.95 to 2.1 reduces the magnitude of $R_{g}$ considerably, i.e., from $R_{g} \simeq 3.8-4.2$ to $R_{g} \simeq 2.50-2.75$ at

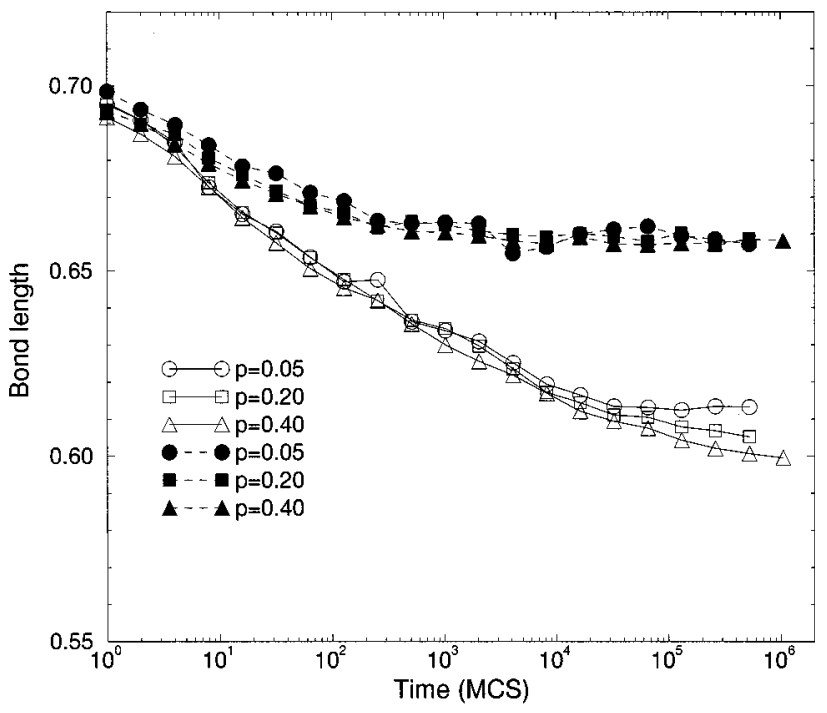

FIG. 9. Bond length vs time for chains of length $L_{c}=40$ at different $p$ and $T=1.0$, with $R_{c}=0.95$ and 2.1.

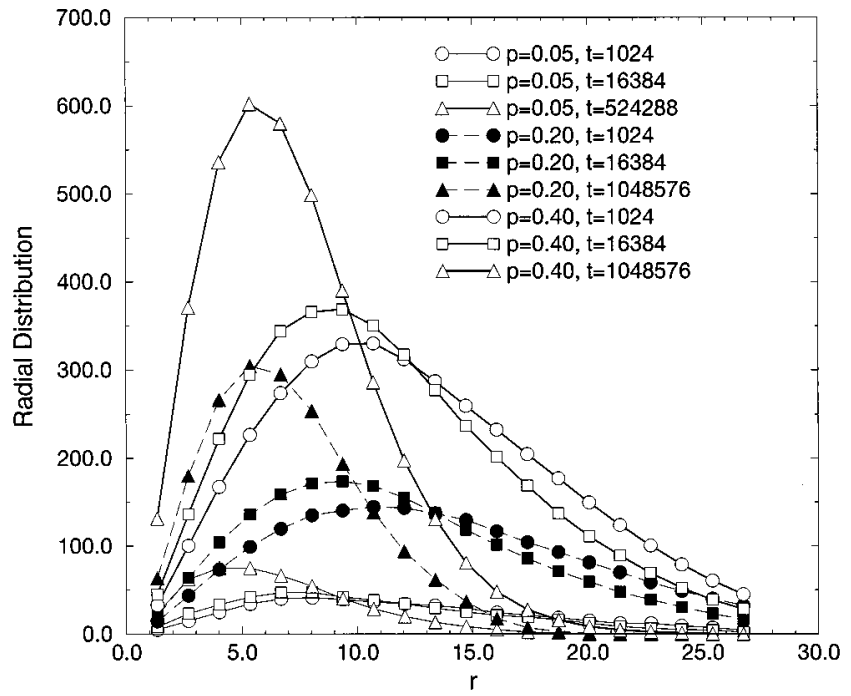

FIG. 10. Variation of the radial distribution function with the distance at different time steps for $L_{c}=80$ at $p=0.05,0.2,0.4$ with $R_{c}=2.1$ and $T=0.2$.

$p=0.05$ and 0.40 . Large $R_{c}$ means long attractive interaction between the chain nodes, which may correspond to a relatively poor quality of solvent for the chain environment. This is consistent with the notion that the size of the chains decreases (collapses) in poor solvent from their extended state in a good solvent.

We evaluate the scaling exponent $(\gamma)$ for the variation of $R_{g}$ with the chain length $\left(L_{c}\right)$,

$$
R_{g} \sim L_{c}^{\gamma} .
$$

Figure 8 shows the variation of $R_{g}$ with $L_{c}$ on a $\log -\log$ scale. The slopes of the linear fit provide an estimate of the exponent $\gamma$ (see Table I). At high temperature $(T=1.0)$, we find $\gamma \simeq 1 / 2$, i.e., a Gaussian conformation with the interaction range $R_{c}=2.1$. Chains seem to stretch a little with $\gamma$ $\simeq 0.63$ at low temperature $(T=0.2)$ with $R_{c}=2.1$ (see Table I) but still the chains conform to random configurations. With the smaller interaction cutoff $\left(R_{c}=0.95\right)$, we find $\gamma$ $\simeq 0.71$ (closer to SAW estimate $3 / 4$ ) at $T=1.0$. However, at low temperature $(T=0.2), \gamma \simeq 1$, i.e., chains are linearly extended (a rodlike conformation on a large scale) with the smaller interaction cutoff. These quantitative measurements of the size of the chains are consistent with our visual inspections of the snapshots.

A typical variation of the average bond length with time is presented in Fig. 9. We see that it takes longer for bonds to relax with larger interaction cutoff $\left(R_{c}=2.1\right)$. The equilibrium bond length $\langle l\rangle$ depends on the range of interaction, $\langle l\rangle$ decreases from about 0.66 with $R_{c}=0.95$ to less than 0.60 at $p=0.4$. Further, we note that the lower the temperature, the smaller the bond length.

\section{Radial distribution}

The radial distribution function $\rho(r)$ is defined as the number of monomers at a distance $r \pm d r$ from the center of mass of the polymer chains. We evaluate $\rho(r)$ in a relatively large range $(r)$ for all parameters we have discussed above. 


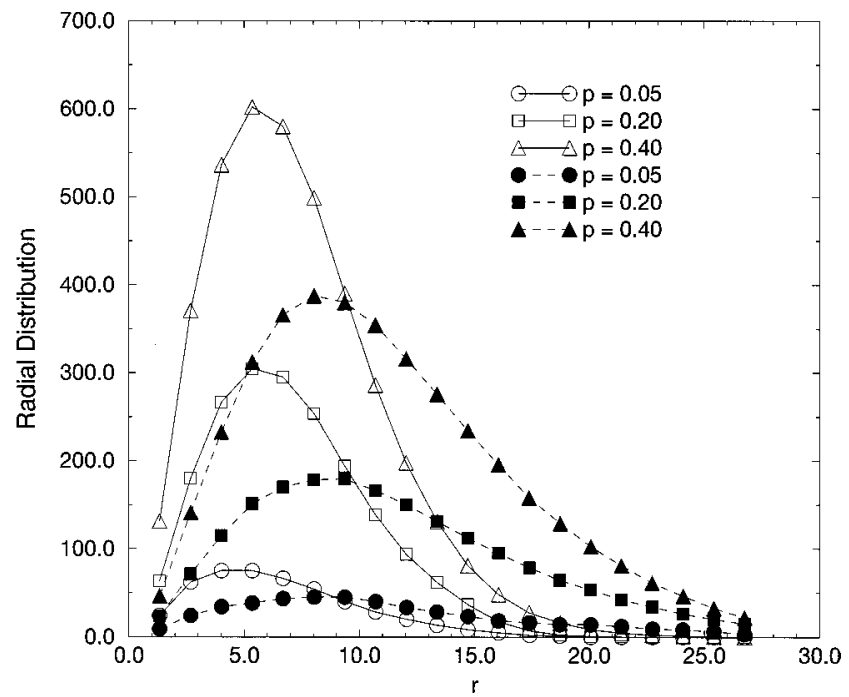

FIG. 11. Radial distribution function vs distance at $p$ $=0.05,0.2,0.4$ for $L_{c}=80$ at $T=0.2$ with $R_{c}=2.1$ (open) and 0.95 (filled).

A typical evolution of $\rho(r)$ is shown in Fig. 10. We see that the peak of the distribution increases and the width reduces in time as the chains segregate into larger aggregates.

The equilibrium distribution function (i.e., the distribution at the end of simulation averaged over independent runs) for $p=0.05,0.20,0.40$ at $T=0.2$ is presented in Fig. 11. We see that the width of the distribution decreases on increasing the polymer concentrations, i.e., the chains become closer to form more compact aggregates at higher $p$. The width of the distribution becomes wider with a relatively long tail with the lower range of interaction $\left(R_{c}=0.95\right)$. In order to see the effect of temperature, we collected the data at low and high temperatures at $p=0.4$ in Fig. 12 . We note that the monomers are relatively more dispersed at higher temperature (longer tails in distribution).

\section{SUMMARY AND CONCLUSION}

A discrete-to-continuum hybrid method is presented, using the efficiency, simplicity, and importance of discrete and continuum simulations to incorporate the appropriate details of small and large scales. In the lattice frame of the host space, we are able to place as many polymer chains as we like, i.e., chains with arbitrarily high concentration, which is not efficient and perhaps not feasible in a continuum beadspring model in a random sequential fashion. Even with the "slithering snake" reptation algorithm alone we are able to prepare an intermediate initial sample with a relatively good random distribution of chains with random SAW conformations using a large-scale athermal stirring. At high temperature $(T=1.0)$, we observe segregation of chains and their clustering with the cutoff $R_{c}=2.1$ of the LJ interaction while there is no segregation with the smaller interaction cutoff $\left(R_{c}=0.95\right)$. This is consistent with the expectation that polymer chains do not mix as well in a relatively poor solvent (ascribed to dominant attractive interaction with $R_{c}=2.1$ ) as in a relatively good solvent $\left(R_{c}=0.95\right)$. At low temperature $(T=0.2)$, on the other hand, we observe a spectacular conformational change to a folded-coil conformation in the long

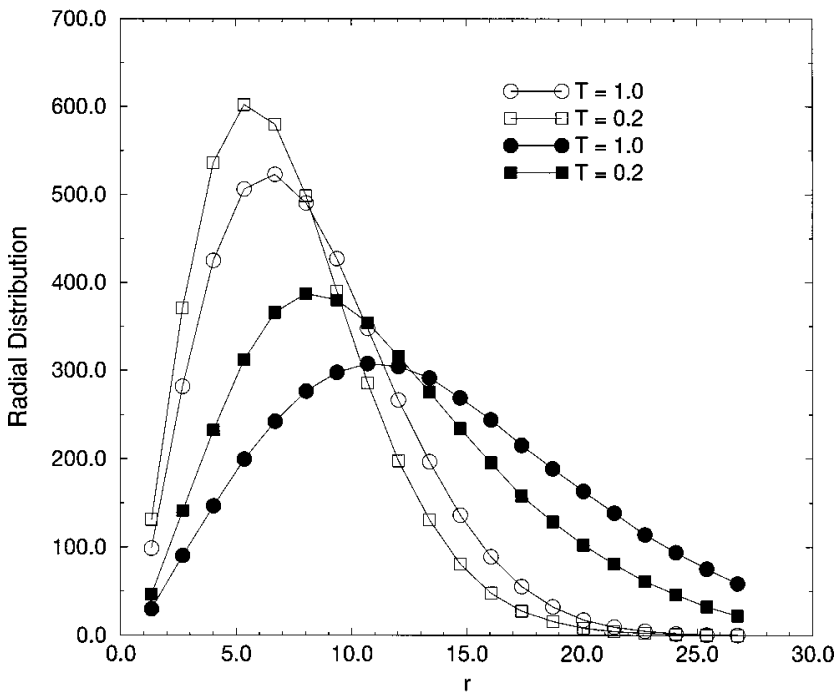

FIG. 12. Radial distribution function vs distance at $T=0.2$ and 1.0 for $L_{c}=80$ at $p=0.4$ with $R_{c}=2.1$ (open) and 0.95 (filled).

time regime; the amplitude of folding depends on the range of interaction, i.e., larger with a larger interaction cutoff.

During the conformational evolution of chains and their segregation or phase separation process, we evaluate the power-law exponents $\left(\nu_{1}, \nu_{2}\right)$ for the rms displacements of the center of mass and their center node, respectively, with time, i.e., $R_{\text {c.m. }} \sim t^{\nu_{1}}, R_{\mathrm{cn}} \sim t^{\nu_{2}}$. We find that these exponents are nonuniversal as they depend on temperature, i.e., $\nu_{1}$ $\simeq 0.40 \quad(T=1.0), 0.30(T=0.2) \quad$ with $R_{c}=2.1$, and $\nu_{1}$ $\simeq 0.45(T=1.0), 0.25(T=0.2)$ with $R_{c}=0.95$ for $L_{c}=40$ chains; these estimates are consistent with other chain lengths (i.e., $L_{c}=80$ ). Thus, the rms displacement of the center of mass of the chains is subdiffusive at low temperature. The exponent $\left(\nu_{2}\right)$ for the center node of the chains also depends on temperature, i.e., $\nu_{2} \simeq 0.30 \quad(T=1.0), 0.10$ $(T=0.2)$ and is nonuniversal. These power-law exponents seem to depend on the range of interaction as well, however, our data is not good enough at present to confirm it conclusively. We would like to point out that such nonuniversal power-law dependence of the rms displacement of chains has been also observed in different context such as chain in a porous medium [15,22].

The radius of gyration $\left(R_{g}\right)$ is generally smaller with the longer range of interaction. The scaling exponent $\gamma\left(R_{g}\right.$ $\sim L_{c}^{\gamma}$ ) exhibits a Gaussian conformation with $R_{c}=2.1$ and extended (SAW) conformation with $R_{c}=0.95$, a relatively good solvent condition. A similar trend is also observed for the average bond length $\langle l\rangle$, which is longer with $R_{c}=0.95$ than that with $R_{c}=2.1$, a relatively poor solvent condition. Radial distribution of monomers (beads) is examined in detail. The variation of the magnitude of the peak of the distribution and its width with temperature and the upper interaction cutoff is consistent with the above observation, i.e., segregation with $R_{c}=2.1$, desegregation with $R_{c}=0.95$ at high temperature $(T=1.0)$, change from a random conformation to a chain folding, etc. We hope to develop and refine this approach further to address more complex issues in polymeric systems. 


\section{ACKNOWLEDGMENTS}

We acknowledge support from a NSF-EPSCoR grant. Simulations were performed at the CADCAM center, Supercomputing and Visualization lab, and on the Linux cluster at the Department of Computational Science, National University of Singapore. Initial stage of program development and test runs with small samples were done at the Mississippi Center for Supercomputing Research.
[1] M. P. Allen and D. J. Tildesley, Computer Simulation of Liquids (Clarendon Press, Oxford, 1996).

[2] D. Frenkel and B. Smit, Understanding Molecular Simulation (Academic Press, New York, 1996).

[3] J. M. Haile, Molecular Dynamics Simulation (John Wiley and Sons, New York, 1992).

[4] H. Gould and J. Tobochnik, An Introduction to Computer Simulation Methods (Addison-Wesley, New York, 1996).

[5] Monte Carlo and Molecular Dynamics Simulations in Polymer Science, edited by K. Binder (Oxford University Press, Oxford, 1995).

[6] Computational Modeling of Polymers, edited by J. Bicerano (Marcel Dekker, New York, 1992).

[7] P. G. de Gennes, Scaling Concepts in Polymer Physics (Cornell University, Ithaca, NY, 1979).

[8] M. Doi and S. F. Edwards, The Theory of Polymer Dynamics (Clarendon, Oxford, 1986).

[9] A. Baumgaertner, in The Monte Carlo Methods in Condensed Matter Physics, edited by K. Binder (Springer-Verlag, New York, 1995).

[10] H. Wendel and J. Noolandi, Macromolecules 24, 1313 (1982).
[11] R. J. Needs, Macromolecules 17, 437 (1984).

[12] J. M. Deutsch, Phys. Rev. Lett. 54, 56 (1985).

[13] K. Kremer, G. S. Grest, and I. Carmesin, Phys. Rev. Lett. 61, 566 (1988).

[14] U. Ebert, A. Baumgaertner, and L. Schaefer, Phys. Rev. Lett. 78, 1592 (1997).

[15] V. Yamakov, D. Stauffer, A. Milchev, G. M. Foo, and R. B. Pandey, Phys. Rev. Lett. 79, 2356 (1997).

[16] P. E. Rouse, J. Chem. Phys. 21, 1273 (1953).

[17] P. H. Verdier and W. H. Stockmayer, J. Chem. Phys. 36, 227 (1962).

[18] F. T. Wall and F. Mandel, J. Chem. Phys. 63, 4592 (1975).

[19] S. Caracciolo and D. A. Sokal, J. Phys. A 20, 2569 (1987).

[20] A. Sokal, in Monte Carlo and Molecular Dynamics Simulations in Polymer Science (Ref. [5]), p. 47.

[21] I. Gerroff, A. Milchev, K. Binder, and W. Paul, J. Chem. Phys. 98, 6526 (1993).

[22] G. M. Foo, R. B. Pandey, and D. Stauffer, Phys. Rev. E 53, 5738 (1996); G. M. Foo and R. B. Pandey, ibid. 55, 4433 (1997). 